\title{
Surgimiento y desarrollo temprano de la ocupación de dietista en la Argentina
}

\section{Emergence and Early Development}

\section{of Dietitian as an occupation in Argentina}

\author{
José Buschini \\ Consejo Nacional de Investigaciones Científicas y Tecnológicas \\ Instituto de Investigaciones en Humanidades y Ciencias Sociales \\ Universidad Nacional de La Plata \\ (Argentina) \\ jbuschini@fahce.unlp.edu.ar
}

Resumen

Este trabajo analiza el surgimiento y el desarrollo temprano de la ocupación de dietista -antecedente inmediato de la actual ocupación de nutricionista- en la Argentina. Se observan los proyectos que dieron lugar a esta nueva ocupación -considerando cómo se concebían los requisitos de formación y las tareas que serían de su incumbencia-, el modo en que se concretaron estos proyectos y el desempeño laboral efectivo de las primeras camadas de dietistas. También, se utilizan herramientas teóricas provistas por la sociología de las profesiones con el fin de interpretar peculiaridades vinculadas con la conformación y el desarrollo inicial de esta ocupación: por un lado, que uno de sus ámbitos de intervención laboral la colocó entonces dentro de la órbita de la medicina y en un plano de subordinación; por otro lado, que en su diseño inicial se tratara de una ocupación restringida a mujeres. Asociado a estas dos características, que su emergencia se debiera a un proyecto impulsado por un médico, ocupación principalmente dominada por hombres.

Palabras Clave: Dietistas - Profesión Médica - Alimentación - Profesionalización - Cierre Social 


\begin{abstract}
This paper analyses the emergence and early development of the dietitian profession -currently known as nutritionist- in Argentina. It focuses on the projects by which this occupation was created -considering the way in which formative requisites and incumbencies were conceived-, the realization of those projects and the access to labor market by the first graduated dietitians. Furthermore, aided by the sociology of professions, this paper aims to understand some peculiarities linked to the creation and early development of this occupation: on the one hand, some aspects of its activities were subordinated to medicine and on the other, its access was restricted to women. In relation to those two characteristics, that the occupation of dietitian was the result of a physician's project, while medicine was mainly a male occupation.
\end{abstract}

Key Words: Dietitian - Medical Profession - Nutrition - Professionalization - Social Closure

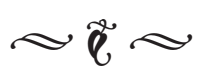

\section{Introducción}

En el año 1935, comenzó a funcionar en la Ciudad de Buenos Aires, la Escuela Municipal de Dietistas. Tres años más tarde, en 1938, obtuvo carácter nacional por un decreto del Poder Ejecutivo Nacional. El establecimiento tenía por fin formar dietistas, una nueva ocupación que ofrecería servicios profesionales a partir de la aplicación de saberes provistos por la dietología, un campo intelectual ${ }^{1}$ que

1.La expresión campo intelectual está tomada en el sentido que le otorga Richard Whitley, para quien constituyen "organizaciones sociales específicas y relativamente bien delimitadas que controlan y dirigen el proceso de investigación en temas particulares". WHITLEY, Richard, La organización social e intelectual de las ciencias, Editorial de la Universidad Nacional de Quilmes, Bernal, 2012, p. 64. Esta decisión permite escapar al debate engorroso sobre el problema de la delimitación de las "colectividades significativas", que incluye diversos modos para referir a los agrupamientos que permiten comprender las orientaciones intelectuales de los investigadores, incluyendo nociones como disciplina, especialidad o áreas de investigación. La pluralidad de conceptos, técnicas y temas que dan forma a la dietología (o ciencias de la nutrición) hace daba sus primeros pasos en la Argentina.

La consolidación de este campo intelectual y la creación de la Escuela de Dietistas acontecieron en un momento en el que la producción, la comercialización y el consumo de los alimentos concitaron la atención de múltiples actores sociales, incluyendo a profesores universitarios, legisladores, funcionarios estatales, empresarios, periodistas y miembros de agrupaciones políticas y de organizaciones de la sociedad civil. Desde distintas plataformas, y en ocasiones de manera polémica, todos ellos se involucraron en la definición de problemas vinculados con la alimentación humana, propusieron soluciones

que no resulte fácil clasificarla con estas etiquetas. Para no abundar en referencias sobre una cuestión marginal a los objetivos del trabajo, un análisis sistemático se encuentra en PREGO, Carlos, "Formación y desarrollo de una tradición científica”, en ALBORNOZ, Marío, KREIMER, Pablo y GLAVICH, Eduardo (editores) Ciencia y Sociedad en América Latina, Editorial de la Universidad Nacional de Quilmes, Bernal, 1996, pp. 493-496. 
y gestionaron recursos para darles curso. Así, a lo largo de la década de 1930 y los primeros años de la década siguiente, se asistió a un proceso en el que, de manera relacionada, diversos temas vinculados con la alimentación humana se constituyeron como objeto de indagación científica y como problema público. Entonces, había razones de peso para que esto fuera así; con sentido contrario, tanto las consecuencias sobre la población de la crisis económica como la posterior expansión del consumo favorecieron el interés por estos temas. Un fenómeno asociado, que no obstante posee independencia analítica, fue la conformación o el afianzamiento de ocupaciones y mercados laborales para atender a cuestiones vinculadas con la alimentación humana. ${ }^{2}$

Este trabajo centra su atención en esta última cuestión, tomando como caso de estudio el surgimiento de la ocupación de dietista, antecedente inmediato de la actual ocupación de nutricionista. Interesará observar los proyectos que dieron lugar a esta nueva ocupación -considerando cómo se concebían los requisitos de formación y las tareas que serían de su incumbencia-, el modo en que se

2. Los procesos que tuvieron lugar en la Argentina se inscribieron en un intenso movimiento en el que organismos internacionales como la Sociedad de Naciones, la Organización Internacional del Trabajo y el Instituto Internacional de Agricultura tuvieron un papel clave. Al respecto, ver BARONA, Josep, The problem of nutrition. Experimental Science, Public Health and Economy in Europe 1914-1945, Peter Lang. International Academic Publishers, Bruselas, 2010, y WEINDLING, Paul, "The role of International Organizations in Setting Nutritional Standards in the 1920s and 1930s", en KAMMINGA Harmke y CUNNINGHAM, Andrew (editors) The science and culture of nutrition, Editions Rodopi, Amsterdam, 1995. En cuanto a los nexos con el caso argentino, ver BUSCHINI, José, "La conformación de la alimentación como problema en la Argentina: el papel de Pedro Escudero y el Instituto Nacional de la Nutrición”, inédito, 2016. concretaron y el desempeño laboral efectivo de las primeras camadas de dietistas. También, se plantearán interrogantes asociados a peculiaridades propias de la conformación de esta ocupación: por un lado, que uno de sus ámbitos de intervención laboral la colocó entonces (situación que persiste) dentro de la órbita de la medicina y en un plano de subordinación; por otro lado, que en su diseño inicial se tratara de una ocupación restringida a mujeres. Asociado a estas dos características, que su emergencia se debiera a un proyecto impulsado por un médico, atributo que remite tanto a su ocupación como a su género.

Las características aludidas permiten inscribir a este caso en un marco más amplio, propio de la sociedad argentina de la primera mitad del siglo XX, que conecta dos procesos paralelos. En primer lugar, la expansión de los alcances de la profesión médica y su creciente injerencia sobre una multiplicidad de esferas sociales. ${ }^{3} \mathrm{Un}$ rasgo destacado de este proceso fue la existencia de un sesgo de género, con un marcado predominio masculino, tanto en lo que refiere a la composición de sus miembros como en lo que toca al acceso a las posiciones de poder $y$

3. Al respecto, ver ARMUS, Diego, "E1 descubrimiento de la enfermedad como problema social", en LOBATO, Mirta (directora) El progreso, la modernización y sus limites, colección Nueva Historia Argentina, T. V, Sudamericana, Buenos Aires, 2000; BELMARTINO, Susana, La atención médica argentina en el siglo XX. Instituciones y procesos, Siglo Veintiuno Editores, Buenos Aires, 2005; BIERNAT, Carolina, "El proceso de centralización del Departamento Nacional de Higiene (1890-1944)", en BIERNAT, Carolina, CERDÁ, Juan Manuel y RAMACCIOTTI, Karina (directores) La salud pública y la enfermería en la Argentina, Universidad Nacional de Quilmes Editorial, Bernal, 2015; GONZÁLEZ LEANDRI, Ricardo, "Itinerarios de la profesión médica y sus saberes de Estado. Buenos Aires, 1850-1910", en PLOTKIN, Mariano y ZIMMERMANN, Eduardo (compiladores) Los saberes del Estado, Edhasa, Buenos Aires, 2012. 
prestigio. ${ }^{4}$ En segundo lugar, la incorporación de mujeres al mercado laboral, en general, y al sector de la salud, en particular. Al respecto, la literatura ha señalado que la participación que en esos años tuvieron las mujeres en el ámbito sanitario fue mayormente subordinada a la profesión médica y se restringió de manera predominante a áreas que respetaban cierta división del trabajo entre géneros: las prácticas de cuidado, la esfera doméstica y tareas que implicaban tomar contacto con otras mujeres, como las que rodeaban a la maternidad. ${ }^{5}$ Así, una conjetura que cabe plantear, aun cuando el alcance empírico de este trabajo no avale conclusiones taxativas, sugiere que existe una conexión estrecha entre ambos procesos, por la cual en sus esfuerzos por expandir los alcances de su ocupación -y, como parte de ello, ganar mercados e influencia en el Estadolos médicos promovieron la incorporación de mujeres al mercado de trabajo de un modo que involucró el control de sus procesos formativos y de su actividad en el contexto laboral.

4. Ver LORENZO, María Fernanda, Que sepa coser, que sepa bordar, que sepa abrir la puerta para ir a la universidad. Las académicas de la Universidad de Buenos Aires en la primera mitad del siglo XX, EUDEBA, Buenos Aires, 2016.

5. LOBATO, Mirta, Historia de las trabajadoras en la Argentina (1869-1960), Edhasa, Buenos Aires, 2007;

MARTIN, Ana Laura, "Mujeres y enfermería: una asociación temprana”, en BIERNAT, Carolina, CERDÁ, Juan Manuel y RAMACCIOTTI, Karina (directores) $L a$ salud pública y la enfermería en la Argentina, Universidad Nacional de Quilmes Editorial, Bernal, 2015; NARI, Marcela, Politicas de la maternidad y maternalismo politico. Buenos Aires, 1890-1940, Biblos, Buenos Aires, 2004; RAMACCIOTTI, Karina y VALOBRA, Adriana, "Feminización y profesionalización de la enfermería (1940-1955)", en BIERNAT, Carolina, CERDÁ, Juan Manuel y RAMACCIOTTI, Karina (directores) $L a$ salud pública y la enfermería en la Argentina, Universidad Nacional de Quilmes Editorial, Bernal, 2015.
El trabajo se encuentra organizado de la siguiente forma. La próxima sección ofrece herramientas conceptuales para analizar el papel de los expertos y el saber experto en la conformación de problemas públicos, los procesos de profesionalización y las especificidades vinculadas a la constitución de una ocupación subordinada a la medicina en aspectos importantes de su actividad e integrada sólo por mujeres. Luego, una sección describe, brevemente, aspectos asociados a la conformación de la alimentación humana como objeto de indagación científica y como problema público en la Argentina durante la década de 1930 y los primeros años de la década siguiente, centrando la mirada en el papel de los médicos. El tratamiento de este tema, lateral en cuanto al objetivo central del trabajo, se justifica debido a que constituye un contexto fundamental para comprender el surgimiento de la ocupación de dietista y algunas de las características que tuvo su desarrollo inicial. Esa cuestión, justamente, es el objeto de la siguiente sección. Por último, en los comentarios finales se recuperan las herramientas conceptuales que fueron presentadas en la segunda sección para plantear una serie de interrogantes y conjeturas sobre las singularidades del caso estudiado.

Antes de dar paso al análisis, cabe realizar una consideración metodológica referida al período considerado, que no tiene fechas que puedan delimitarlo con exactitud. Por ello, en más de una oportunidad se hará referencia a la década de 1930 y a la primera mitad de la década de 1940 , sin mayores precisiones. Esto se debe, en primer lugar, a que no hay un momento exacto en el que se puedan fechar las intervenciones públicas que referían a la necesidad de dar nacimiento a la ocupación de dietista. Antes bien, se trata de proyectos que acompañaron 
el establecimiento de la dietología en el país, que tímidamente comenzó a aparecer a fines de la década de 1920 y cobró gran fuerza en la década siguiente. En segundo lugar, a que si bien la ocupación se siguió desarrollando luego de mediados de la década de 1940, aquí se ha trabajado con fuentes documentales que no van más allá de ese período. Por fuera de estas razones, se debe también a que no interesa tanto realizar una descripción cronológica de acontecimientos sino dar cuenta de las características centrales del proceso que tuvo lugar en esos años.

\section{Saber experto, profesiones y género: herramientas conceptuales para el análisis del surgimiento de la ocupación de dietista}

Como se indicó, la emergencia de la ocupación de dietista en la Argentina ocurrió en un contexto en el que diversas cuestiones asociadas a la producción, la comercialización y el consumo de los alimentos se erigieron a la vez como objeto de indagación científica y como problema público. Con base en desarrollos conceptuales elaborados por Magali Sarfatti Larson, se considera aquí que en ese momento esos temas cobraron la forma de campos discursivos con unidad ideológica temática. ${ }^{6}$ Esta autora realiza este aporte en el marco de sus esfuerzos por comprender el papel del conocimiento experto en relación con el fenómeno profesional, este último eje de su trayectoria académica, y su preocupación gira alrededor del modo en que el conocimiento es producido y aplicado de modo tal que la vida

6. SARFATTI LARSON, Magali, "Acerca de los expertos y los profesionales o la imposibilidad de haberlo dicho todo", en Revista de Educación, Madrid, 1990, número extraordinario, pp. 200-225. de las personas queda afectada. Como parte de esta tarea, se vale de las obras de Michel Foucault y Pierre Bourdieu para dar forma a la noción de campos discursivos con unidad ideológica temática, a los que presenta como escenarios en los que se pone en juego la capacidad para hablar de manera autorizada sobre asuntos circunscritos y, como resultado de estas intervenciones, para producir efectos sobre dominios de prácticas. Analizando los procedimientos que, según Foucault, controlan la producción del discurso, realiza una lectura sociológica del tercer tipo de procedimientos identificados por este autor -aquellos que guardan relación con el control del acceso a su producción y circulación- y llega por esa vía a argumentar que los temas que logran constituirse como campos discursivos con unidad ideológica temática generan dos tipos de disputas: entre expertos, por un lado, y entre expertos y legos, por otro lado. Es clave, para que las luchas en un campo se estructuren de esta manera, que los legos reconozcan el carácter diferencial de la pericia que los expertos se atribuyen, algo que en las sociedades contemporáneas se ve facilitado por el lugar que ocupa la ciencia y es asegurado mediante la difusión a gran escala del sistema educativo gratuito y la emisión permanente de mensajes desde los medios de comunicación de masas. Establecer o no el carácter obligatorio de pasteurizar la leche, señalar que importantes sectores de la población se encontraban mal alimentados o desnutridos y formular propuestas para revertir esta situación, apoyar o cuestionar la comercialización de café torrado -procedimiento por el que se agrega azúcar al tostar los granos de café-, promover la creación de organismos estatales para favorecer la producción de algún tipo de alimento $\mathrm{y}$ sostener la necesidad de crear un código 
alimentario nacional, son todos ejemplos a propósito del modo en que, en el período bajo estudio, ciertos temas fueron objeto de disputa para una pluralidad de actores, quienes en sus intervenciones enlazaron argumentos técnicos, económicos, políticos y morales.

En paralelo a la conformación de campos discursivos con unidad ideológica temática en torno de la alimentación humana, tuvo lugar un fenómenoque, pese a guardar estrecha conexión, posee independencia analítica: las necesidades asociadas a la aplicación práctica del cuerpo de conocimientos científicos que sirvieron de base a las intervenciones expertas sobre el tema generaron iniciativas para el establecimiento de nuevas ocupaciones o, cuando ya existían, para fortalecer su lugar dentro de mercados laborales. Esto alude a una cuestión que posee una marcada centralidad para la sociología de las profesiones, conceptualizada bajo el rótulo de profesionalización, que capta su carácter procesual. Aun con notables diferencias de interpretación, tanto para el enfoque estructural-funcionalista como para las corrientes que lo cuestionaron desde diferentes ángulos resulta clave entender cómo una ocupación deviene profesión, ya se considere que es la adquisición de algo inherente a esas ocupaciones (como en el caso del estructural-funcionalismo, que ponía el énfasis en el entrenamiento técnico y los valores) o de algo que otros actores les imputan, un estatus que se obtiene mediante una acción colectiva, eminentemente política, por la que se interpela con éxito al Estado y a la sociedad en pos de obtener privilegios, como un monopolio dentro de un mercado o autonomía técnica en el trabajo. Por fuera de sus particularidades, la literatura involucrada en los cuestionamientos a la visión del estructural-funcionalismo, a la que se sigue en este trabajo, ha demostrado la existencia de rasgos característicos en estos procesos, como el control de los establecimientos educativos en donde se forman los nuevos miembros de la profesión -por añadidura, la regulación de la oferta de mano de obra al mercado laboral, mediante el otorgamiento de certificados habilitantes para el ejercicio de la profesión-, la realización de acciones colectivas orientadas a obtener prerrogativas por parte del Estado-típicamente incluyen la creación de asociaciones profesionales y la sanción de códigos de ética- y la persuasión de la población a partir de enfatizar la importancia y la calidad de los servicios ofrecidos. ${ }^{7}$

El surgimiento de la ocupación de dietista y la definición de sus incumbencias, por tanto, puede entenderse en el marco de proyectos que apuntaron a delimitar las condiciones de acceso a mercados laborales -en vías de constitución- en torno de problemáticas suscitadas por la alimentación humana. Como

7. Ver FREIDSON, Eliot, La profesión médica. Un estudio de sociología del conocimiento aplicado, Ediciones Península, Barcelona, 1978[1970]; SARFATTI LARSON, Magali, The Rise of Profesionalism, Berkeley, Los Angeles and London, 1977. Miradas de conjunto muy esclarecedoras sobre el desarrollo de la sociología de las profesiones se encuentran en ABBOTT, Andrew, The System of Professions. An Essay on the Division of Expert Labor, The University of Chicago Press, Chicago, 1988, pp. 3-20; BELMARTINO, Susana, Nuevas reglas de juego para la atención médica en la Argentina ¿QQuién será el árbitro?, Lugar editorial, Buenos Aires, 1999, pp. 77-147; GONZÁLEZ LEANDRI, Ricardo, La construcción histórica de una profesión. Asociación e instituciones médicas en Buenos Aires: 1852-1895, Tesis doctoral, Universidad Complutense de Madrid, 1997, pp. 2-58. Una revisión bibliográfica que busca articular el desarrollo de este campo con los estudios sobre género en POZZIO, María, "Análisis de género y estudios sobre profesiones: propuestas y desafíos de un diálogo posible -y alentador-", en Revista Sudamérica, Mar del Plata, 2012, No 1, pp. 102-129. 
fue señalado, hubo dos rasgos destacados en el surgimiento de esta ocupación. Por un lado, que en importantes aspectos de su actividad quedó subordinada a la medicina. Por otro lado, que estuvo restringida a mujeres. Asociado a estas dos cuestiones, que su emergencia no fue impulsada por sus destinatarias sino por un médico. Para pensar esta especificidad, un primer aporte relevante lo constituye el trabajo de Eliot Freidson, a propósito de las relaciones entre la profesión médica y el personal paramédico, que agrupa a las "ocupaciones cuyo trabajo está a la vez organizado en torno a las tareas sanitarias y controlado, en última instancia, por la autoridad médica". ${ }^{8}$ Este control, sostiene, se manifiesta de cuatro modos: "en primer lugar, gran parte del conocimiento técnico adquirido por el personal paramédico en el curso de su aprendizaje y utilizado en el curso de su trabajo es generalmente revelado, ampliado y aprobado por médicos. En segundo lugar, las tareas realizadas por el personal paramédico suelen servir de ayuda, y no de sustitución directa, a las tareas primordiales del diagnóstico y el tratamiento. En tercer lugar, el personal paramédico actúa de una manera subordinada por cuanto que su trabajo se realiza de ordinario a petición o por "orden" de médicos que a menudo lo supervisan. Por último, el prestigio asignado por el público en general a las ocupaciones paramédicas es menor que el asignado a los médicos". ${ }^{9}$

Un segundo aporte significativo son los desarrollos conceptuales y la evidencia empírica provistos por Anne Witz, quien estudia procesos de profesionalización que involucran

8. FREIDSON, Eliot, "Personal paramédico", en Sills, David (editor) Enciclopedia Internacional de las Ciencias Sociales, Aguilar, Madrid, V. VIII, 1976, pp. 147-152.

9. Ídem, p. 147. a mujeres. ${ }^{10}$ En particular, algo que interesa a los fines de este trabajo, es el uso que hace de la teoría del cierre social y el modo en que explora las potencialidades asociadas a incorporar una perspectiva de género en esta teoría. Elaborada inicialmente por Frank Parkin a partir de ideas contenidas en la obra de Max Weber -vinculadas al modo en que ciertos grupos sociales intentan ampliar sus recompensas limitando el acceso a recursos y oportunidades a un grupo restringido de candidatos, con la consecuencia añadida de excluir a otros grupos del acceso a estas recompensas-, esta teoría plantea la existencia de dos grandes mecanismos de acción social colectiva mediante los que se apunta a maximizar la adquisición de recompensas y oportunidades: exclusión -pretensión por parte de un grupo de asegurarse una posición privilegiada a expensas de otros grupos mediante un proceso de subordinación, generando una categoría de rechazados o extraños- y usurpación -la respuesta que dan los grupos subordinados para obtener una participación mayor en los recursos-.${ }^{11}$ En las sociedades modernas, plantea Parkin, existen dos dispositivos centrales mediante los que se produce el cierre social excluyente: las instituciones que rodean a la propiedad (entendida en tanto control de los medios de producción) y las calificaciones y méritos académicos o profesionales. Este último, habitualmente designado como credencialismo, es el que adquiere relevancia aquí y sobre el que gira el trabajo de Witz. Según Parkin, este fenómeno alude al "uso exagerado de los títulos educativos como medio de controlar la entrada a posiciones

10.WITZ, Anne, Professions and Patriarchy, Taylor and Francis e-Library, New York, 2004[1992].

11. PARKIN, Frank, Marxismo y Teoría de Clases. Una critica burguesa, Espasa-Calpe, Madrid, 1984, pp. 70-71. 
clave en la división del trabajo". ${ }^{12}$

Para Witz, quien recupera este enfoque neoweberiano pero también perspectivas neomarxistas, los proyectos profesionales -a los que concibe como aquellas estrategias por las cuales una ocupación apunta a "establecer un monopolio sobre la provisión de destrezas y competencias en un mercado de servicios"deben ser entendidos como proyectos de cierre social ocupacional que tienen un contenido de género asociado y son posibles en el marco de una estructura social particular, a la que define como capitalismo patriarcal. ${ }^{13}$ Esta enunciación, demasiado amplia, adquiere especificidad mediante una elaboración conceptual que busca refinar la teoría del cierre social, de modo tal que pueda captar el modo en que las estrategias de cierre social proveen "medios para movilizar al poder masculino con el fin de reclamar para sí recursos y oportunidades que son distribuidos mediante el mecanismo del mercado de trabajo". ${ }^{14}$ En este marco, da cuenta de diferentes tipos de estrategias, una de las cuales es especialmente relevante en el marco de este artículo.Inspirada por los trabajos de Freidson sobre personal paramédico, de Kreckel sobre estrategias de cierre social demarcatorio y de Larkin sobre imperialismo ocupacional en la división médica del trabajo, explora la importancia de las estrategias de cierre social demarcatorio -que se relacionan con los intentos de supervisar y regular el trabajo de ocupaciones con las que se mantienen vínculos dentro de la división del trabajo- desde una perspectiva

\section{2. Ídem, p. 83.}

13. WITZ, Anne, Professions and patriarchy..., Op. Cit., p. 61.

14. Ídem, p. 42.

142 de género. ${ }^{15}$ Considera que son cruciales para la creación y el sostenimiento de relaciones de género desiguales dentro de una jerarquía ocupacional en el mercado de trabajo, puesto que no se orientan a excluir a las mujeres sino a circunscribir su participación a actividades o esferas de competencia que se encuentran en una relación de subordinación con respecto a ocupaciones dominadas por hombres. ${ }^{16}$

Como se indicó, Witz conecta este enfoque neoweberiano con perspectivas neomarxistas. En este sentido, toma nota de debates internos dentro de la teoría del cierre social, en particular las críticas a propósito del excesivo énfasis en la agencia y el consecuente descuido del peso asignado a la estructura social -en tanto facilita o inhibe el acceso a los recursos que permiten concretar proyectos colectivos-, y señala que, en los casos que ella analizó, el éxito de las estrategias de cierre social impulsadas por los médicos -ya sean las de exclusión o las de carácter demarcatorio- se vio facilitado por el modo en que las instituciones de la sociedad civil expresaban relaciones de poder que favorecían a un tipo de actor social particular: el hombre burgués. Aun cuando Witz se encarga de situar empíricamente el alcance de esta afirmación -los casos que analiza acontecen en la sociedad inglesa del siglo XIX y los primeros años del siglo XX-, no parece fuera de lugar extrapolar estas consideraciones al objeto bajo estudio en este trabajo. Según

15. FREIDSON, Eliot, “Personal...”, Op. Cit.;

KRECKEL, Reinhard, “Unequal Opportunity Structure and Labour Market Segmentation”, en Sociology, Londres, 1980, V. XIV, No 4, pp. 525-550; LARKIN, Gerald., "The Medical Dominance in Britain: Image and Historical Reality", en The Milbank Quarterly, New York, 1988, V. LXVI, Suplement 2, pp. 117-132.

16. WITZ, Anne, Professions and patriarchy..., Op. Cit., p. 45 . 
indica Mirta Lobato, a fines del siglo XIX, se consolidó en la Argentina una división de roles entre los géneros por la cual, como tendencia, los hombres dominaron la esfera pública y la actuación de las mujeres quedó circunscrita dentro del espacio doméstico. ${ }^{17}$ Específicamente en lo que refiere al ámbito sanitario, las tribunas desde las que se podía dar curso a proyectos de expansión de la profesión médica y controlar la actividad de ocupaciones cercanas, como el parlamento nacional, la Academia Nacional de Medicina y los cargos directivos en universidades y oficinas sanitarias estatales, estaban ocupadas, exclusivamente, por hombres. Existe, por cierto, una agrupación que introduce una nota disonante en esta interpretación: la Sociedad Nacional de Beneficencia. Bajo cierta mirada, estas damas de la élite social, que tuvieron una marcada influencia en el ámbito sanitario argentino durante el período bajo estudio, fueron una amenaza o un escollo para las "ambiciones imperiales" de la profesión médica; o al menos para ciertos sectores dentro de ella. ${ }^{18}$ No obstante, su accionar colectivo no implicó proyectos de profesionalización ni tuvo injerencia en ellos, puesto que sus fuentes de legitimación eran otras. En este sentido, clase y género se entrecruzaban, pues no eran estas mujeres de la élite quienes quedaban comprendidas bajo los proyectos, promovidos por médicos hombres, de incorporación subordinada al mercado de trabajo.

17. LOBATO, Mirta, Historia de trabajadoras..., Op. Cit., p. 13.

18. Sobre el accionar de las damas de beneficencia, especialmente en lo que refiere a las tensiones con la profesión médica, ver BELMARTINO, Susana, $L a$ atención médica..., Op. Cit., pp. 52-59; RAMACCIOTTI, Karina, La politica sanitaria del peronismo, Biblos, Buenos Aires, 2009, pp. 25-29.

\section{La conformación de la alimentación humana como objeto científico y problema público en la Argentina: el papel de los expertos}

En el proceso de conformación de la alimentación humana como objeto científico y como problema público en la Argentina, fue clave el papel de algunos grupos profesionales (médicos, bromatólogos, veterinarios, ingenieros agrónomos, entre otros), quienes estudiaron cuestiones relativas a la producción, la comercialización y el consumo de los alimentos, señalaron la existencia de problemas a los que se debía dar respuesta y buscaron articular su actividad con otros actores sociales con el fin de dar curso a los proyectos que elaboraron para intervenir sobre estas realidades. Por fuera de las peculiaridades vinculadas con sus adscripciones profesionales, todos ellos compartían rasgos que son relevantes para el análisis. Se trataba de personas que tenían un desempeño laboral ligado a la docencia universitaria y al quehacer científico-técnico -ya sea en la propia universidad o en oficinas estatales- y que poseían vínculos con políticos, funcionarios estatales y personalidades destacadas de la sociedad civil, que se reforzó en esos años por el interés que despertaban sus conocimientos y destrezas de cara a las necesidades de un Estado que extendía sus alcances y de una incipiente industria alimentaria.

Dentro de este cuadro, el caso de los médicos interesa a los fines de este trabajo puesto que conecta directamente con el establecimiento de la ocupación de dietista. Aquí, fue un actor particular, Pedro Escudero -asistido por un grupo estable de colaboradores que comenzó a 
trabajar con él a medida que contó con recursos para garantizar su desarrollo laboral-, quien ganó el centro de la escena. ${ }^{19}$ La medicina se encontraba entonces plenamente consolidada como profesión liberal y como cuerpo experto del Estado, sus miembros ocupaban los diferentes organismos oficiales creados con el fin de atender a las necesidades sanitarias de la población y, tras haber conseguido éxitos importantes en el control de las enfermedades infectocontagiosas, impulsaban proyectos destinados a paliar enfermedades crónicas (cáncer, dolencias cardiovasculares, las propias enfermedades de la nutrición) y los 1lamados "males sociales" (alcoholismo, enfermedades venéreas). Esta acumulación colectiva fue una plataforma para Escudero quien, a comienzos de los años veinte, tenía una posición sólida dentro del ámbito médico-universitario porteño, que incluía los cargos de Profesor Titular de la cátedra de Clínica Médica (medular en la formación profesional) en la Facultad de Ciencias Médicas de la Universidad de Buenos Aires y de Jefe de Servicio en el Hospital Rawson, perteneciente a la incipiente red de hospitales públicos de la Capital Federal. Además, gozaba de reconocimiento por sus múltiples publicaciones y desde joven había sido un activo participante en los grupos que pretendían renovar las prácticas formativas dentro de la escuela profesional. Con estos antecedentes en su haber, buscó conseguir algo que, tal como muestra la literatura, parecía ser entonces la cúpside consagratoria para los médicos ligados

19. Esta sección presenta de manera estilizada elementos de un trabajo propio a propósito del papel del médico Pedro Escudero y el Instituto Nacional de la Nutrición en la conformación de la alimentación como objeto científico y como objeto de políticas públicas en la Argentina. Ver BUSCHINI, José, "La conformación de la alimentación...", Op. Cit. a la actividad docente: dirigir un instituto de investigación, un espacio más preocupado por ampliar el conocimiento asociado a ciertas disciplinas científicas o especialidades médicas que por realizar tratamientos de rutina. ${ }^{20}$ Así, en el año 1924 gestionó ante Abel Zubizarreta, director de la Asistencia Pública Municipal -el organismo sanitario de la Ciudad de Buenos Aires-, la creación en el Hospital Rawson del Instituto de Enfermedades de la Nutrición, que comenzó a funcionar cuatro años más tarde, en 1928. A lo largo de la década de 1930, este establecimiento creció notablemente. En el año 1934, por medio de una ley nacional, obtuvo fondos que le permitieron conseguir su autonomía con respecto del Hospital Rawson. Cuatro años más tarde, en 1938, un decreto del Poder Ejecutivo Nacional le otorgó carácter nacional, colocándolo con el nombre de Instituto Nacional de la Nutrición bajo dependencia del Ministerio de Relaciones Exteriores y Culto. En esos años, fue adquiriendo su forma definitiva y delimitando el alcance de sus actividades, que al momento de su nacionalización incluían la atención médica, la asistencia social, la investigación científica, la provisión de servicios técnicos al Estado, la formación profesional y la educación popular. Este crecimiento fue paralelo al del interés público por la alimentación humana, que entre otras cosas, se expresó en la sanción de leyes y decretos que consideraban aspectos relacionados con la asistencia alimentaria de la población, como los que en los años 1934, 1936 y 1938 dieron origen, respectivamente, a la Junta Nacional de Ayuda al Niño, la

20. Ver BUSCHINI, José y ZABALA, Juan Pablo, "La medicina experimental en la Argentina durante la primera mitad del siglo XX: las trayectorias de Ángel Roffo, Salvador Mazza y Bernardo Houssay", en Revista Brasileira da História da Ciência, San Pablo, 2015, V. VIII, № 1, pp.22-38. 
Dirección de Maternidad e Infancia y la Comisión Nacional de Ayuda Escolar.

Para presentar de manera simplificada un proceso que tiene una complejidad mayor, sobre todo en lo que refiere a la temporalidad de las influencias, lo que ocurrió entonces habla de un refuerzo mutuo por el cual los saberes de la dietología permitieron designar y cuantificar fenómenos (por ejemplo, la desnutrición de sectores de la población) que ya preocupaban a actores sociales con capacidad para definir políticas públicas, esto favoreció el otorgamiento de recursos para desarrollar investigaciones en el área de la alimentación humana, las investigaciones resultantes permitieron identificar nuevos fenómenos o realizar propuestas de intervención sobre determinadas realidades (por ejemplo, elaboración de tablas de composición química de alimentos disponibles en el país, encuestas sobre consumo alimentarios de la población trabajadora, cálculo de la relación entre ingresos de la familia trabajadora y el costo de los alimentos) y esto incidió sobre la formulación e implementación de nuevas políticas (por ejemplo, la creación de organismos provinciales destinados a cuestiones referidas a la alimentación de la población).

En el marco de este trabajo, hay un aspecto vinculado con este proceso de refuerzo mutuo que reviste especial interés, por su significado analítico: las pretensiones de los miembros del Instituto de la Nutrición para constituirse como voces autorizadas en materia de alimentación humana se sustentaban en un conjunto de saberes que ofrecían criterios nutricionales científicos con los cuales se podía elaborar regímenes dietéticos que, según sus promotores, garantizaban una salud óptima con el menor costo posible, cuestión que adquiría expresión sintética bajo el rótulo de alimentación racional. En particular, tres conjuntos de saberes relativamente delimitados dentro de la dietología resultaban fundamentales: técnica dietética, arte coquinario y economía dietética. ${ }^{21}$ La primera, basada en disciplinas como la física y la química, aludía a los procedimientos utilizados para confeccionar un régimen dietético, sea para el hombre sano o para el hombre enfermo, e incluía tanto un examen minucioso de los alimentos y las transformaciones a los que estos eran sometidos durante su cocción como, en el caso del hombre enfermo, la consideración de la fisiopatología del órgano u órganos enfermos, los síntomas y síndromes concomitantes, el momento evolutivo de la enfermedad y el estado del aparato digestivo. Arte coquinario, por su parte, refería a la forma de preparar los alimentos y las recetas que se podían elaborar de acuerdo a los alimentos disponibles y a los requisitos de la técnica dietética. En ambos casos, adquirían centralidad las leyes de la alimentación, etiqueta con la que Escudero sistematizó (y se apropió selectivamente de) los conocimientos disponibles a nivel internacional sobre las cuestiones que debían ser consideradas para elaborar un régimen

21. Para presentaciones de las características de cada uno más extensas que la que aquí se realiza, ver BUSCHINI, JOSÉ, "La conformación de la alimentación como problema...”, Op. Cit.; ESCUDERO, Pedro, "Las leyes de la alimentación", en Trabajos y publicaciones del Instituto Nacional de la Nutrición, Buenos Aires, 1938, V. II, T.I, pp. 25-48; ESCUDERO, Pedro y PIERÁNGELI, Enrique, "La economía dietética en la enseñanza oficial de la Escuela Nacional de Dietistas. Su orientación y finalidad", en Trabajos y publicaciones del Instituto Nacional de la Nutrición, Buenos Aires, 1938, V. II, T. I, pp. 152154; ESCUDERO, Pedro y ROTHMAN, Boris, "La técnica dietética. Su orientación y finalidad”, en Trabajos y publicaciones del Instituto Nacional de la Nutrición, Buenos Aires, 1938, V. II, T.I, pp. 183-189. 
dietético "libre de todo error", ${ }^{22}$ y en torno de las cuales tenían lugar nociones como las de valor calórico total; balance; régimen suficiente, insuficiente, generoso y excesivo; enflaquecimiento; desnutrición; entre otras. Por último, para lograr que las exigencias de la técnica dietética fuesen cumplidas con el costo mínimo -fundamental en un momento en que el gasto en alimentación constituía el elemento más importante dentro del presupuesto de la familia media-, la economía dietética permitía analizar el precio de los alimentos en el mercado en relación con su valor nutritivo (una actividad importante dentro del INN (Instituto Nacional de la Nutrición) fue la elaboración de tablas de composición química de los alimentos disponibles en el país) y sobre esta base considerar su inclusión en regímenes dietéticos.

\section{Alimentación humana y mercados laborales: el caso de la dietista}

Entre sus múltiples iniciativas, los actores del ámbito académico involucrados en la conformación de la alimentación humana como objeto científico y como problema público buscaron crear o expandir mercados laborales asociados a sus destrezas, mediante la obtención de prerrogativas por parte del Estado y la persuasión de la población. Para ello, resultaron centrales la creación de especialidades o carreras en establecimientos educativos de nivel superior y los reclamos al Estado para que regule las incumbencias de las ocupaciones asociadas a estos títulos y garantice la contratación de las personas que contaran

22. ESCUDERO, Pedro, “Las leyes de...”, Op. Cit., p. 32. 146 con ellos. ${ }^{23}$ Esto fue complementado con la difusión de conocimientos a la población, a través de los medios de comunicación de masas, la realización de charlas en organizaciones de la sociedad civil y la incorporación de contenidos en la educación de nivel inicial y medio. Sin negar la importancia que estas actividades pudieron tener para ofrecer a la población herramientas que le permitieran mejorar sus niveles de salud o prevenir el fraude, constituyeron un mecanismo a través del cual los profesionales apuntaron a que se reconozca un saber experto $-\mathrm{y}$ a las personas que lo dominaban; es decir, a ellos mismosque prescribía prácticas alrededor de las que comenzaban a conformarse mercados laborales y se definían políticas públicas. En el caso de los médicos, Escudero -secundado por sus colaboradores- fue una vez más quien encarnó públicamente estas demandas. En intervenciones públicas realizadas a lo largo de la década de 1930 y la primera mitad de la década siguiente, señaló la importancia de formar especialistas en el tema y delimitar sus

23. Según Sarfatti Larson, la creación de carreras en establecimientos educativos de nivel superior tiene un lugar estratégico en los proyectos de profesionalización, pues constituyen ámbitos en los que logran articularse dos elementos que suelen evolucionar de manera independiente: un cuerpo de conocimientos relativamente abstracto, susceptible de aplicación práctica, y un mercado. Allí, en efecto, se dan los procesos de entrenamiento en los que, con alta estandarización cognitiva, se "producen los productores" y, por la despersonalización que esto supone -en tanto la presentación ante la clientela que a partir de entonces realizará el profesional no descansa tanto en sus rasgos individuales como en su papel de representante de un colectivo que domina prácticamente un saber esotérico, aplicable a la resolución de problemas-, el producto que ofrecen se vuelve reconocible para los públicos potenciales. SARFATTI LARSON, Magali, The rise of..., Op. Cit., pp. 40-47. 
incumbencias laborales. ${ }^{24}$ También, presentó proyectos ante legisladores nacionales $\mathrm{y}$ autoridades universitarias para que esto tenga curso efectivo.

En sus intervenciones, Escudero argumentó que el médico que quisiera especializarse en el campo de las enfermedades de la nutrición debía estudiar contenidos de dietología, y que las necesidades asociadas a la administración de alimentos a los enfermos había obligado a crear una nueva ocupación, la de dietista. Para dar un sentido concreto a lo que implicaba esta última, ofrecía una evaluación de los dos modelos que a su juicio se encontraban disponibles a nivel internacional, el estadounidense y el europeo -del que decía que se podía tratar como conjunto pese a las diferencias entre países-, considerando tres criterios que permitían establecer una comparación: los conocimientos previos exigidos para ingresar en las escuelas de formación de dietistas, el plan de estudio y los programas de enseñanza, y la duración de los estudios. Tras analizar las diferencias que existían en relación con estos criterios, se inclinaba por el modelo estadounidense,

24.Ver ESCUDERO, Pedro, "Dietólogos, dietistas y cocineros", en ESCUDERO, Pedro, Alimentación. Colaboraciones publicadas en La Prensa, Hachette, Buenos Aires, 1934, pp. 87-93; ESCUDERO, Pedro, "La política nacional de la alimentación en la República Argentina”, en Trabajos y publicaciones del Instituto Nacional de la Nutrición, Buenos Aires, 1939, V.III, pp. 22-26; ESCUDERO, Pedro, La dietista argentina. Su orientación médico social, Instituto Nacional de la Nutrición, Buenos Aires, 1942; ESCUDERO, Pedro, "La obra cultural y económico-social de los médicos dietólogos y dietistas graduadas en el Instituto Nacional de la Nutrición (de 1935 a 1944)", en Revista de la Asociación Argentina de Dietología, Buenos Aires, 1945, V. III, No 10 pp. 83-127; LÓPEZ LEÓN, Arturo, "Discurso en representación del curso de Médico Dietólogo, 14 de octubre de 1939”, en Tercera Conferencia Internacional de la Alimentación. Boletin Suplementario, Instituto Nacional de la Nutrición, Buenos Aires, 1939, pp. 5-9. puesto que suponía una formación de tipo universitario y un desempeño laboral con funciones y responsabilidades propias. En el caso europeo, por el contrario, no pasaba de ser un colaborador de hospital sin gran formación al que equiparaba con personal de enfermería. Sobre esta base, postulaba que entre el médico dietólogo y la dietista existía una relación de complementación comparable a la que se daba con otras ocupaciones que gravitaban en torno de la profesión médica (como las de farmacéutico, dentista y bioquímico), por la cual ambos constituían una unidad funcional. ${ }^{25}$ Esto era así debido a que, por un lado, compartían conocimientos que les garantizaban un lenguaje común para trabajar de manera conjunta, pero, por otro lado, cada uno de ellos poseía conocimientos específicos. De esta forma, sólo el médico dietólogo estaba capacitado para diagnosticar y prescribir un tratamiento dietético al enfermo, mientras que sólo la dietista conocía en profundidad los fundamentos de técnica dietética, arte coquinario y economía dietética, lo que la habilitaba para ejecutar la prescripción solicitada por el médico. Esta presentación de la dietología y de las ocupaciones a que daba lugar, por cierto, era incompleta, pues sólo refería a la alimentación del hombre enfermo. Sin embargo, los problemas que en esos años suscitaba la alimentación humana y los saberes que la dietología ofrecía para dar respuestas prácticas a ellos permitían a su

25. La forma en que Escudero presentaba la comparación resulta interesante porque asume como natural algo que, desde la perspectiva que guía este trabajo, es el resultado de procesos mediante el cual las ocupaciones se involucran en la búsqueda de obtener la propia autonomía y/o la subordinación de otras ocupaciones. Los casos escogidos por Escudero, justamente, han mostrado a lo largo de su historia y en diferentes contextos nacionales relaciones cambiantes con la profesión médica. 
impulsor imaginar un campo de intervención más amplio, especialmente para las dietistas, que incluía no sólo la alimentación del hombre enfermo (en hospitales, asilos, sanatorios, profesión civil) sino también la alimentación de personas sanas (en colegios, clubes, comedores de talleres, oficinas, restaurantes, hoteles, comedores escolares), la enseñanza oficial (en colegios, escuelas, facultad de medicina) y popular (mediante la radiodifusión, el periodismo gráfico, conferencias públicas y la asistencia social), y el desempeño en asociaciones comerciales e industriales (realizando propaganda vinculada a la venta de productos alimentarios).

Un elemento central a propósito del modo en que Escudero concebía la ocupación de dietista, cuyo significado no resulta del todo fácil desentrañar, consiste en la restricción de la actividad a mujeres. Al respecto, daba dos argumentos. En primer lugar, que esto era así en los modelos foráneos que había estudiado. Se trataba, decía, de una "corriente universal". ${ }^{26}$ En segundo lugar, con mayor importancia sustantiva, por razones vinculadas a un modo de entender la distribución de roles entre los géneros, por la cual “a pesar de la versación y de la profundidad de los estudios de las dietistas, la alimentación tiene como eje de su realización la cocina, rincón del hogar” y, en este marco, "la mujer aporta, con sus conocimientos, ${ }^{27}$ la ductilidad de su espíritu, las maneras suaves y el ambiente hogareño que la acercan a los seres que sufren”. ${ }^{28}$ Sin negar la importancia o la veracidad de estos fundamentos -a nivel

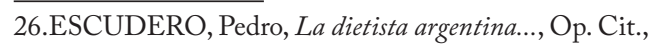
p. 20.

27. Esta formulación es ambigua pero en el contexto parece querer indicar "junto con".

28. Ibídem.

148 internacional, efectivamente, la ocupación se encontraba fuertemente feminizada; en el ámbito sanitario argentino, como se dijo, se había consolidado una división del trabajo por la que el papel de la mujer se restringía a prácticas de cuidado, al ámbito doméstico y al tratamiento de problemas que implicaban contacto con otras mujeres-, cabe dejar asentado, como interrogante para indagaciones ulteriores, si tal vez no pesaron otras razones al momento de imprimirle este sesgo de género a la ocupación, las cuales quizás no resultaba tan fácil explicitar públicamente. En particular, la competencia que en esos mismos años pudieron suponer otras mujeres en el ámbito que se quería conquistar, como la Sociedad Nacional de Beneficencia, con su siempre señalada influencia en los servicios sanitarios, o las visitadoras de higiene, otra ocupación vinculada con la medicina que tenía injerencia en la esfera doméstica y presentaba una composición predominantemente femenina. También de esos años, cabe recordar, es la emergencia de una figura como la de Petrona Carrizo de Gandulfo ("Doña Petrona”), quien a través de la masividad que le ofrecían la radio y la prensa gráfica comenzó a gravitar en la conformación de las costumbres culinarias argentinas. $^{29}$

29. Sobre la influencia creciente de Petrona a lo largo de la década de 1930, ver: PITE, Rebekah, Creating a Common Table in Twentieth-Century Argentina. Doña Petrona, Women, E Food, The University of North Carolina Press, Chapel Hill, 2013. Al respecto, es muy ilustrativo, un caso que muestra esta autora. En el año 1933, la Junta Nacional de Carnes, en sus esfuerzos por promover hábitos culinarios que permitieran comercializar cortes de carne poco utilizados, contrató a Escudero, quien creó la Comisión para el Estudio y Propaganda de la Carne y propuso un "enfoque científico" para realizar esta tarea. Algunos años más tarde, en 1939, Escudero fue reemplazado por Doña Petrona, quien tenía un estilo para comunicar que, sin ser científico, era considerado más eficaz. Ídem, p. 85-87. 
Las consideraciones reseñadas estuvieron en la base de los proyectos que, desde los primeros años de la década de 1930, Escudero comenzó a presentar ante autoridades universitarias y estatales con el fin de crear la carrera de dietista y la especialidad de médico dietólogo. El primer logro en esa dirección fue la creación, en el año 1933, de la Escuela Municipal de Dietistas, que comenzó a funcionar dos años más tarde bajo la órbita del Instituto Municipal de la Nutrición y que en el año 1938 fue nacionalizada junto con el establecimiento. El plan de estudios de esta carrera, para cumplir con los diferentes ámbitos de intervención que se imaginaban, se estructuró en dos ciclos en los que se estudiaban aspectos técnicos y económicos de la alimentación del hombre sano y del hombre enfermo, con una duración total de tres años. En sintonía con el campo laboral anhelado, los contenidos específicos incluían conocimientos de anatomía, fisiología y patología de distintos grupos poblacionales (adultos, niños, mujeres embarazadas, recién nacidos), dietoterapia para cada uno de estos grupos poblacionales, estudio químico y tecnológico de los alimentos, economía dietética, técnica dietética, y arte coquinario. Una vez concluidos los estudios, se realizaba una prueba final de idoneidad por la que las alumnas obtenían un diploma que las acreditaba como dietista y les permitía ejercer la profesión en cuanto a "la prescripción, realización y vigilancia de la alimentación de las personas sanas, individual y colectivamente consideradas; la realización y la vigilancia de la alimentación de los enfermos que están bajo el control directo de un médico o de una institución médica [;] la dirección y trabajo en oficinas técnicas, públicas o privadas, que aborden cuestiones económicas, comerciales o industriales en relación con los alimentos y la alimentación [y] la enseñanza en las escuelas y colegios de toda asignatura en relación con la biología, la higiene y la economía doméstica de los alimentos y de la alimentación". ${ }^{30}$ Estas incumbencias fueron reconocidas primero por un decreto del Poder Ejecutivo Nacional, del año 1938, y luego confirmadas en el año 1944, cuando se sancionó la ley de asociaciones profesionales. La ocupación de dietista, así, quedó incluida dentro de las profesiones liberales reglamentadas, adquiriendo personería técnica.

Junto con la creación de la Escuela de Dietistas y el reconocimiento legal de las incumbencias de la nueva ocupación, Escudero consiguió que la especialidad de dietólogo adquiera carta de ciudadanía en la formación médica. En 1936 y en 1938, respectivamente, se crearon en la Facultad de Ciencias Médicas de la Universidad de Buenos Aires la Cátedra de Clínica de la Nutrición (con Escudero como Profesor Titular, comenzó a funcionar en el año 1937 con sede en el INN y un curso especializado de Médico Dietólogo, en un momento en que también se discutieron y reglamentaron otras especialidades médicas. La cátedra, destinada a alumnos que cursaran el último año de la carrera de medicina, tenía clases teóricas y prácticas sobre alimentación normal y enfermedades de la nutrición, incluyendo en este último caso la asistencia a pacientes. La especialidad de médico dietólogo, por su parte, era un ciclo de dos años al que podían acceder médicos diplomados y que tenía un contenido similar al de la escuela

30. ESCUDERO, Pedro, "La política nacional...", Op. Cit., p. 25. Junto a esto, se estableció que las enfermeras recibidas en escuelas oficiales que realizaran un aprendizaje práctico durante un semestre escolar en un Servicio de Alimentación del INN y que rindieran de manera satisfactoria la prueba correspondiente recibirían el diploma de enfermeras auxiliares idóneas en alimentación. 
de dietistas, con excepción de los contenidos destinados a impartir nociones básicas de medicina y de aquellos centrados en economía dietética, técnica dietética y arte coquinario. En el primer caso, por razones obvias; en los otros dos, de manera coherente con los modos en que eran pensados los roles de cada una de estas ocupaciones, en tanto el médico prescribía pero no ejecutaba.

En la segunda mitad de la década de 1930, por tanto, en el país comenzaron a formarse dietistas y médicos dietólogos. Algunos datos sistematizados por Escudero en el año 1945, aun cuando constituyen una fuente documental de alcance limitado, permiten realizar comentarios sobre el desarrollo inicial de estas carreras y sobre la inserción laboral temprana de las dietistas. ${ }^{31}$ En primer lugar, en lo que refiere a la matrícula y los niveles de graduación. Entre 1935 y 1944, se inscribieron quinientos noventa y ocho alumnas en la Escuela de Dietistas y doscientos cincuenta y nueve alumnos al curso de dietólogo. De ellos, entre 1938 y 1944, se graduaron ciento cuatro dietistas -trece de ellas, en realidad, eran auxiliares en alimentación, un título intermedio- y ciento tres médicos dietólogos. En su mayoría, los inscriptos procedían de la ciudad de Buenos Aires, aunque también realizaron la carrera y se graduaron un número importante de personas de diferentes provincias del país y de países limítrofes. Esto último se vio favorecido por becas que otorgó el gobierno nacional, establecidas como parte del creciente interés que generaban los problemas vinculados con la alimentación humana y de la posición que consiguió la dietología en ese marco. La inscripción y graduación de estos estudiantes del interior del país y de otros

31. ESCUDERO, Pedro, “La obra cultural...", Op. Cit. países (de los médicos dietólogos graduados, quince eran becarios extranjeros y quince becarios del interior; de las dietistas graduadas, cincuenta y dos eran becarias del interior y nueve eran becarias extranjeras) redundó en la posterior expansión de la dietología, pues la beca exigía el compromiso de un posterior desempeño laboral en la provincia por la que era otorgada, en el caso de los dietólogos y dietistas argentinos, o que se garantice al graduado un cargo público referente a la alimentación, en el caso de los dietólogos y dietistas de otros países. En segundo lugar, en cuanto al desempeño laboral en esos primeros años en que se establecieron esas ocupaciones. Específicamente en relación con las dietistas, los datos ofrecidos por Escudero muestran que veintiuna de las cuarenta y una dietistas de la ciudad de Buenos Aires ocupaban cargos en el Estado, cuatro lo hacían en clínicas y hospitales particulares, y dieciséis ejercían la profesión de manera privada. Por su parte, de las cincuenta y una dietistas del interior del país, veintisiete tenían cargos públicos, ocho trabajaban en clínicas y hospitales particulares, y dieciséis ejercían la profesión de manera privada. ${ }^{32}$

Avanzando hacia una descripción cualitativa, y siempre enfatizando el carácter limitado de la evidencia documental disponible, es posible caracterizar algunos aspectos de los cargos públicos, los únicos sobre los que se poseen datos. En la ciudad de Buenos Aires, el propio Instituto de la Nutrición fue el principal canal de contratación. Allí, a mediados de la década de 1940, cinco dietistas trabajaban bajo la dirección de un médico dietólogo en

32. La discrepancia con el número total de graduadas, de ciento cuatro, se debe a que tres dietistas habían abandonado la actividad profesional tras contraer matrimonio y a que, nueve de ellas, eran becarias extranjeras. 
la Secretaría Técnica -sección dependiente de la Dirección General-, en la tarea de elaborar respuestas a las consultas técnicas que eran remitidas al establecimiento por parte de instituciones públicas y privadas; catorce dietistas se desempeñaban bajo la dirección de cuatro médicos dietólogos en la Sección de Alimentación, en actividades que incluían la investigación, la enseñanza y la organización de servicios alimentarios; y, por último, una dietista asistía a una médica dietóloga -la única mujer que en esos años contaba con esa especialización- en los laboratorios de biología, con la función de planear la alimentación de los animales que se utilizaban para los experimentos. Las dietistas participaban también en el importante servicio de prensa del establecimiento, por medio del cual se realizaban las campañas de difusión de conocimiento a la población, tanto en los programas de radio que se emitían por Radio del Estado (de las cinco conversaciones semanales, tres estaban a su cargo) como en la elaboración de los artículos que eran remitidos a diferentes periódicos del país. En las diferentes provincias del país, por su parte, los Estados fueron también canales importantes de contratación. En la primera mitad de la década de 1940, algunas provincias crearon -o se encontraban en vías de crear- dependencias públicas destinadas a estudiar el problema de la nutrición e intervenir sobre él, y para ello contrataron a especialistas formados en el Instituto de la Nutrición. En Mendoza, se creó el Departamento de Nutrición. Allí, cuatro dietistas trabajaban bajo la dirección de un médico dietólogo. En Santa Fe, se creó una Sección de Nutrición dentro del Departamento de Sanidad, dependiente del Ministerio de Salud Pública y Trabajo. Para esta oficina fueron contratados un médico dietólogo y una dietista, con la dirección del primero. En la provincia de Buenos Aires, en la órbita de la Dirección de Salubridad, se creó una Subsección de Nutrición dentro de la Sección Médica. Al igual que en el caso de Santa $\mathrm{Fe}$, trabajaron de manera conjunta un médico dietólogo y una dietista, con el mismo esquema jerárquico. En las provincias de San Luis y Corrientes, por último, estaban previstas creaciones similares, en donde también serían contratados médicos dietólogos y dietistas.

Tanto en el Instituto de la Nutrición como en las dependencias creadas por las diferentes provincias, hay dos elementos compartidos en relación con la actividad de las dietistas. El primero de ellos concierne a la tarea docente, ya se trate de la formación profesional o de la educación popular. En relación con el primer caso, dos dietistas se desempeñaron como Jefe de Trabajos Prácticos en la Cátedra de Alimentación del niño normal y sano del Curso de Médicos Dietólogos; y otra tuvo a su cargo la Cátedra de Nutrición del tercer Curso de Visitadoras de Higiene, que se dictaba en el Instituto de Higiene de esa casa de estudios. En relación con el segundo caso, tanto en la ciudad de Buenos Aires como en el resto del país las dietistas dictaron cursos y charlas de distinta duración en organizaciones de la sociedad civil. El segundo elemento refiere al hecho de que las dietistas siempre trabajaron bajo la supervisión de médicos dietólogos, aun cuando las actividades que desempeñaban no concernían a la alimentación del hombre enfermo, únicas en las que la subordinación entre ocupaciones se encontraba reglamentada. En relación con esto, otro ámbito suma evidencia a propósito del carácter subordinado de la ocupación. En el año 1942, cuando ya existían una importante cantidad de médicos dietólogos y dietistas, fue creada la Asociación Argentina de Dietología, 
pensada a la vez como una sociedad científica (creó una revista anexa, en la que se difundían investigaciones realizadas en el Instituto Nacional de la Nutrición y breves noticias que referían a la situación de la dietología en otros países) y de defensa de los intereses profesionales. Allí, los cargos más importantes eran ocupados por médicos dietólogos y las dietistas sólo aparecían en cargos de vocales. Es difícil pensar, que en una asociación con estas características, las dietistas pudieran expresar allí eventuales conflictos interocupacionales o canalizar proyectos en pos de su autonomía.

Para finalizar, tres situaciones descritas por Escudero permiten dar cuenta de otros aspectos vinculados con el desarrollo laboral inicial de las dietistas. La primera de ellas concierne a dos casos que contradicen la tendencia general sobre el carácter subordinado de la dietista con respecto de los médicos dietólogos. En la provincia de Córdoba, una dietista fue colocada al frente de la Inspección de comedores escolares y colonias de vacaciones y contó con la asistencia de otra dietista. Allí, tuvo a su cargo la organización del servicio de alimentación de ciento veintitrés establecimientos. En la provincia de San Juan, una dietista fue designada como directora técnica de los comedores escolares. Las otras dos situaciones dan cuenta de algunos límites en los intentos de la dietología (y de las dietistas especialmente) para ocupar mercados laborales que se consideraban como propios. Por un lado, en los referidos comedores escolares, algo que se volvía relevante en el marco de la creación por parte del Estado de la Comisión Nacional de Ayuda Escolar. Según Escudero, este era un cargo que, por sus conocimientos, correspondía a una dietista. Sin embargo, este no era el caso a mediados de la década de 1940. Para Escudero, la mala reglamentación de la ley que había dado lugar a la creación de la Comisión Nacional de Ayuda Escolar había derivado en la ocupación de los puestos por parte de un personal sin la formación adecuada ("desde visitadoras sociales, que nada tienen que ver con la ciencia de la alimentación, hasta semianalfabetas, sin más bagaje intelectual que lo aprendido en la escuela elemental") y, aun en los casos en que fueron contratadas dietistas, indicaba que sus sugerencias no habían sido implementadas. ${ }^{33}$ Por otro lado, un campo de intervención anhelado por los impulsores de la dietología que, al menos en los años que se consideran aquí, no se desarrolló: el de la organización de comedores en empresas privadas. Pese a que se habían recibido consultas al respecto, finalmente, sólo se había contratado los servicios ofrecidos por los especialistas en una ocasión.

\section{Comentarios finales}

En este trabajo se analizó la emergencia y el desarrollo inicial de la ocupación de dietista. Por lo expuesto, es posible afirmar que este proceso comparte rasgos con otras ocupaciones que se crearon o expandieron en esos años, como la de médico dietólogo o la de bromatólogo. En un momento en que la alimentación humana concitó gran atención para una multiplicidad de actores sociales, la necesidad de resolver problemas vinculados con la producción, la comercialización y el consumo de los alimentos favoreció la expansión de mercados laborales asociados a las ocupaciones que ofrecían servicios técnicos relacionados con esos temas. Es así como se puede entender la relativa facilidad con que

33. Ídem, p. 119. 
Escudero consiguió los apoyos necesarios para crear la Escuela de Dietistas, los éxitos en materia de la matrícula, las prerrogativas que obtuvo esta ocupación por parte del Estado y la capacidad de las egresadas para acceder al mercado de trabajo.

Por fuera de esta característica compartida, aparecen las especificidades. En este sentido, los tres rasgos más destacados, fuertemente conectados entre sí, fueron que el proyecto de creación de esta ocupación se debió a un actor externo a su futuro desarrollo -un médico-, la restricción a mujeres y la subordinación de importantes aspectos de la actividad a la profesión médica. En relación con los primeros dos rasgos, se indicaron los esfuerzos de los médicos por expandir los alcances de su ocupación y se planteó como conjetura -que la evidencia empírica disponible no alcanzó a responder- que promover la incorporación al mercado de trabajo de mujeres con un alto nivel de calificación constituyó una forma de lograr el dominio sobre esferas sociales tradicionalmente ocupadas por mujeres, como el espacio doméstico. Sean o no estas las razones, lo que sí se pudo apreciar a partir de la evidencia disponible es que tanto la forma en que se concibió la nueva ocupación como su desarrollo inicial presentaron en importantes aspectos de la actividad características muy cercanas a las que Freidson identificó como propias del personal paramédico, con una marcada subordinación con respecto de la profesión médica, manifiesta en el control de los procesos formativos, en la reglamentación de las incumbencias laborales y en el propio ámbito de trabajo. No obstante, la forma en que fue concebida la ocupación contempló también márgenes de autonomía para tareas que eran consideradas dominio propio de la dietología, como la organización de comedores para colectividades sanas, la docencia y el trabajo para empresas y comercios. De igual modo, se pudo observar que, aun para pocos casos, esto tuvo concreción efectiva. Estas consideraciones constituyen un punto de partida para profundizar el análisis, tanto en lo que refiere al período estudiado como al desarrollo posterior de la ocupación, atendiendo especialmente a procesos de construcción de identidad colectiva por parte de las dietistas, a las posturas asumidas en relación con el lugar que les asignó la profesión médica dentro de la división del trabajo y a las iniciativas para conquistar espacios de autonomía y nuevos mercados.
Recibido: 25 - 06 - 2016
Aceptado: 19 - 10 - 2016
Publicado 30 - 12 - 2016 


\section{Bibliografía}

ABBOTT, Andrew, The System of Professions. An Essay on the Division of Expert Labor, The University of Chicago Press, Chicago, 1988.

ARMUS, Diego, "El descubrimiento de la enfermedad como problema social", en LOBATO, Mirta (directora) El progreso, la modernización y sus limites, Editorial Sudamericana, Buenos Aires, 2000.

BARONA, Josep, The problem of nutrition. Experimental Science, Public Health and Economy in Europe 19141945, Peter Lang. International Academic Publishers, Bruselas, 2010.

BELMARTINO, Susana, Nuevas reglas de juego para la atención médica en la Argentina ¿¿uién será el árbitro?, Lugar Editorial, Buenos Aires, 1999.

BELMARTINO, Susana, La atención médica argentina en el siglo XX. Instituciones y procesos, Siglo Veintiuno Editores, Buenos Aires, 2005.

BIERNAT, Carolina, "El proceso de centralización del Departamento Nacional de Higiene (1890-1944)", en BIERNAT, Carolina, CERDÁ, Juan Manuel y RAMACCIOTTI, Karina (directores) La salud pública y la enfermería en la Argentina, Universidad Nacional de Quilmes Editorial, Bernal, 2015.

BUSCHINI, José, "La conformación de la alimentación como problema en la Argentina: el papel de Pedro Escudero y el Instituto Nacional de la Nutrición”, inédito, 2016.

BUSCHINI, José y ZABALA, Juan Pablo, "La medicina experimental en la Argentina durante la primera mitad del siglo XX: las trayectorias de Ángel Roffo, Salvador Mazza y Bernardo Houssay", en Revista Brasileira da História da Ciência, San Pablo, 2015, V. VIII, No 1, pp. 22-38.

ESCUDERO, Pedro, "Dietólogos, dietistas y cocineros", en ESCUDERO, Pedro, Alimentación. Colaboraciones publicadas en La Prensa, Hachette, Buenos Aires, 1934, pp. 87-93.

ESCUDERO, Pedro, "Las leyes de la alimentación”, en Trabajos y publicaciones del Instituto Nacional de la Nutrición, Buenos Aires, V. II, T.I, pp. 25-48.

ESCUDERO, Pedro, "La política nacional de la alimentación en la República Argentina”, en Trabajos y publicaciones del Instituto Nacional de la Nutrición, Buenos Aires, Buenos Aires, 1939, V. III, pp. 1-143.

ESCUDERO, Pedro, La dietista argentina. Su orientación médico social, Instituto Nacional de la Nutrición, Buenos Aires, 1942.

ESCUDERO, Pedro, "La obra cultural y económico-social de los médicos dietólogos y dietistas graduados en el Instituto Nacional de la Nutrición (de 1935 a 1944)", en Revista de la Asociación Argentina de Dietologia, Buenos Aires, 1945, V. III, No 10, pp. 83-127.

ESCUDERO, Pedro y PIERÁNGELI, Enrique, "La economía dietética en la enseñanza oficial de la Escuela Nacional de Dietistas. Su orientación y finalidad", en Trabajos y publicaciones del Instituto Nacional de la Nutrición, Buenos Aires, 1938, V.II, T.I, pp. 152-154. 
ESCUDERO, Pedro y ROTHMAN, Boris, "La técnica dietética. Su orientación y finalidad”, en Trabajos y publicaciones del Instituto Nacional de la Nutrición, Buenos Aires, 1938, V. II, T. I, pp. 183-189.

FREIDSON, Eliot, La profesión médica. Un estudio de sociología del conocimiento aplicado, Ediciones Península, Barcelona, 1978 [1970].

FREIDSON, Eliot, "Personal paramédico", en SILLS, David (editor) Enciclopedia Internacional de las Ciencias Sociales, Madrid, 1976, V. VIII, Aguilar, pp. 147-152.

GONZÁLEZ LEANDRI, Ricardo, La construcción histórica de una profesión. Asociación e instituciones médicas en Buenos Aires: 1852-1895, Tesis doctoral, Universidad Complutense de Madrid, 1997.

GONZÁLEZ LEANDRI, Ricardo, "Itinerarios de la profesión médica y sus saberes de Estado. Buenos Aires, 1850-1910”, en PLOTKIN, Mariano y ZIMMERMANN, Eduardo (compiladores) Los saberes del Estado, Edhasa, Buenos Aires, 2012.

LARKIN, Gerald, "The Medical Dominance in Britain: Image and Historical Reality", en The Milbank Quarterly, New York, 1988, V. LXVI, Suplement 2, pp. 117-132.

LOBATO, Mirta, Historia de las trabajadoras en la Argentina (1869-1960), Edhasa, Buenos Aires, 2007

KRECKEL, Reinhard, "Unequal Opportunity Structure and Labour Market Segmentation”, en Sociology, Londres, 1980, V.IV, No 4, pp. 525-550.

LÓPEZ LEÓN, Arturo, "Discurso en representación del curso de Médico Dietólogo, 14 de octubre de 1939”, en Tercera Conferencia Internacional de la Alimentación. Boletín Suplementario, Instituto Nacional de la Nutrición, Buenos Aires, 1939, pp. 5-9.

LORENZO, María Fernanda, Que sepa coser, que sepa bordar, que sepa abrir la puerta para ir a la universidad. Las académicas de la Universidad de Buenos Aires en la primera mitad del siglo XX, EUDEBA, Buenos Aires, 2014.

MARTIN, Ana Laura, “Mujeres y enfermería: una asociación temprana”, en BIERNAT, Carolina, CERDÁ, Juan Manuel y RAMACCIOTTI, Karina (directores) La salud pública y la enfermería en la Argentina, Universidad Nacional de Quilmes Editorial, Bernal, 2015; NARI, Marcela, Politicas de la maternidad y maternalismo politico. Buenos Aires, 1890-1940, Biblos, Buenos Aires, 2004.

NARI, Marcela, Politicas de la maternidad y maternalismo politico. Buenos Aires, 1890-1940, Biblos, Buenos Aires, 2004.

PARKIN, Frank, Marxismo y Teoría de Clases. Una crítica burguesa, Espasa-Calpe, Madrid, 1984.

PITE, Rebekah, Creating a Common Table in Twentieth-Century Argentina. Doña Petrona, Women, E̋ Food, The University of North Carolina Press, Chapel Hill, 2013.

POZZIO, María, "Análisis de género y estudios sobre profesiones: propuestas y desafíos de un diálogo posible -y alentador-", en Revista Sudamérica, Mar del Plata, 2012, No 1, pp. 102-129. 
PREGO,Carlos, "Formación y desarrollo de una tradición científica", en ALBORNOZ, Marío, KREIMER, Pablo y GLAVICH, Eduardo (editores) Ciencia y Sociedad en América Latina, Editorial de la Universidad Nacional de Quilmes, Bernal, 1996.

RAMACCIOTTI, Karina, La politica sanitaria del peronismo, Biblos, Buenos Aires, 2009.

RAMACCIOTTI, Karina y VALOBRA, Adriana, "Feminización y profesionalización de la enfermería (1940-1955)”, en BIERNAT, Carolina, CERDÁ, Juan Manuel y RAMACCIOTTI, Karina (directores) La salud pública y la enfermería en la Argentina, Universidad Nacional de Quilmes Editorial, Bernal, 2015.

SARFATTI LARSON, Magali, The Rise of Profesionalism, University of California Press, Berkeley, Los Angeles and London, 1977.

SARFATTI LARSON, Magali, "Acerca de los expertos y los profesionales o la imposibilidad de haberlo dicho todo", en Revista de Educación, Madrid, 1990, número extraordinario, pp. 200-225.

WEINDLING, Paul, "The role of International Organizations in Setting Nutritional Standards in the 1920s and 1930s", en KAMMINGA, Harmke y CUNNINGHAM, Andrew (editors) The science and culture of nutrition, Editions Rodopi, Amsterdam, 1995.

WHITLEY, Richard, La organización social e intelectual de las ciencias, Editorial de la Universidad Nacional de Quilmes, Bernal, 2012.

WITZ, Anne, Professions and Patriarchy, Taylor and Francis e-Library, New York, 2004[1992]. 